\title{
Generation of the wheat mutants with high iron (Fe) and expression analysis of the responsive genes involved in iron accumulation and ethylene response
}

$\mathrm{Na} \mathrm{Li}$

Qingdao Agricultural University

Yuetong Li

Qingdao Agricultural University

Jiawei Sun

Qingdao Agricultural University

Xinying Sui

Qingdao Agricultural University

Yanchong Yu

Qingdao Agricultural University

Chun-Hai Dong ( $\square$ chunhai79@yahoo.com )

Qingdao Agricultural University https://orcid.org/0000-0003-0568-0300

\section{Research Article}

Keywords: wheat, Iron (Fe), gene expression, transcriptome, ethylene, TaERF

Posted Date: January 27th, 2022

DOl: https://doi.org/10.21203/rs.3.rs-1259888/v1

License: (c) (i) This work is licensed under a Creative Commons Attribution 4.0 International License.

Read Full License 


\section{Abstract}

Wheat (Triticum aestivum L.) is one of the most important staple crops, feeding one-third of the global population. Among the mineral nutrients, iron $(\mathrm{Fe})$ is one of the necessary trace elements for human body. Iron deficiency will lead to various diseases. Although some progress has been made on the $\mathrm{Fe}$ biofortification aimed at increasing the levels of the essential mineral in the staple crop, little is known about the molecular regulations of the Fe accumulation-associated gene expressions in the wheat plant. In the present study, two wheat mutant lines with high ferric contents were identified. Transcriptomic analysis indicated that the differentiated expression genes (DEGs) were involved in different biological processes such as catalytic reaction, translation, biologic regulations, biosynthesis of secondary metabolites, and stress signaling. Further study revealed that the responsible genes for Fe homeostasis such as TaFIBP (encoding a ferric ion binding protein), TaZIP29 (a ZRT/IRT-like protein (ZIP) encoding gene) and TaYSL 1 (wheat homologue of the Arabidopsis yellow stripe-like protein encoding gene) were highly induced when treated with exogenous Fe in the wheat mutant lines. In addition, it was observed that the ethylene sensitivity of the wheat mutant lines with high iron were obviously altered. And, the transcript levels of the ethylene responsive genes (TaERF1, 2, 3, 4, 6) were significantly affected in the wheat mutant lines, suggesting that ethylene may participate in regulation of the iron accumulation in wheat. All these results advance our understanding of the molecular regulations in the Fe accumulation of wheat.

\section{Introduction}

Iron is one of the necessary trace elements for human body. Iron deficiency will lead to malnutrition, decreased body immunity, retardation of growth, and other diseases (Briat et al. 2015). In plants, iron participates in various biological processes such as chlorophyll synthesis, mitochondrial respiration, nitrogen assimilation, production and scavenging of reactive oxygen species (ROS), and enzymatic activities (Hansch and Mendel 2009). Fe deficiency dramatically affects plant growth, formation, and productivity. Plants absorb Fe from soil. In soil, iron availability is often low due to its low solubility. To adapt to Fe-deficient conditions, plants have developed multiple strategies to absorb Fe. Strategy I occurs in non-graminaceous monocots and dicots, while strategy II exists in graminaceous monocots (Römheld and Marschner, 1986; Kobayashi and Nishizawa, 2012). When exposed to a Fe-deficient environment, the Strategy I plants usually promote the Fe absorbing by enhanced ferric reductase activity, acidification of the rhizosphere and up-regulation of $\mathrm{Fe}^{2+}$ transporters (Curie and Briat, 2003; Hell and Stephan, 2003; Romera and Alcántara, 2004), while the Strategy II plants often increased the secretion of phytosiderophores (PSs) to form a Fe ${ }^{3+}-\mathrm{MA}$ complex (Kobayashi and Nishizawa, 2012).

In past years, numerous studies had identified the responsible genes in Fe assimilation and transportation. For example, it was found that AtYSL 1 (a member of the YELLOW STRIP-LIKE family) participates in Fe loading and transport in Arabidopsis (Le Jean et al. 2005; Waters et al. 2006; Chu et al. 2010). In addition, it was reported that AtOPT3, a member of the oligo-peptide transporter family, also regulates seed Fe accumulation in Arabidopsis. Lack of AtOPT3 causes decreased seed Fe accumulation 
(Stacey et al. 2008). In plants, ferritins were thought to sequester Fe, and buffer against the excess of Fe (Roschzttardtz et al. 2013).

Plant hormones and small molecular regulators play important roles in responses to iron deficiency (Yang et al., 2013). For instance, auxin, brassinosteroids, and gibberellins (GAs) and others have been reported to be involved in the regulation of Fe deficiency-induced physiological processes in plants. Several lines of evidence suggested that auxin is involved in plant root growth and the expression of Fe-uptake genes under Fe deficiency condition (Jin et al., 2011; Giehl et al., 2012; Ivanov et al., 2012; Rai et al., 2015). Qi et al (2012) reported that OsARF12, a transcription factor that activates the auxin response gene, affected Fe accumulation and distribution in rice. Xu et al (2014) provided evidence indicating that OsABCB14 is involved in both auxin transport and Fe homeostasis in rice. In addition, Liu et al (2015) reported that auxin signaling is involved in the regulation of Fe-deficiency induced impairment of photosynthetic activity and shoot growth in rice. Brassinosteroids are also involved in Fe homeostasis in rice (Wang et al., 2015). In a GA-deficient Arabidopsis mutant, expression of the genes associated with Fe-deficiency responses was up-regulated (Matsuoka et al., 2014). Wild et al (2016) reported that the spatial distribution of GA-regulated DELLA growth repressors in Arabidopsis roots was adapted to the root system architecture and the Fe-uptake machinery to the plant's Fe demand. Recently, Wang et al (2017) provided evidence supporting that GA was in mediation of Fe homeostasis by negatively regulating $\mathrm{Fe}$ transport and translocation in rice. Moreover, a study showed that jasmonate responsive genes were also up-regulated in rice when subjected to Fe deficiency (Kobayashi et al., 2016).

The phytohormone ethylene also participated in the regulation of Fe accumulation in plants. Early studies suggested that ethylene may not be involved in the regulation of Fe-deficiency responses by strategy II plants (Romera et al., 1999; Romera and Alcántara, 2004). Wu et al (2011) showed that ethylene regulates expression of the genes associated with Fe acquisition in rice plants. In Arabidopsis, it was reported that EIN3 can physically interact with and stabilize FIT1 (Lingam et al. 2011). The Arabidopsis AtERF72 negatively regulates Fe-deficiency responses by binding to the promoters of several Fe-deficiency responsive genes (Liu et al. 2017). In addition, it was found that the rice ERF genes are transcriptionally regulated under Fe overload stress conditions (dos Santos et al. 2013). A recent study showing that lack of ERF95 or EIN3 reduces seed Fe accumulation in Arabidopsis, while overexpression of ERF95 could rescue Fe accumulation in the ein3 loss-of-function mutant (Sun et al. 2020).

Bread wheat (Triticum aestivum L.) is cultivated on more land than any other crop. So far, most studies have been focused on the Fe biofortification aimed at increasing the levels of the essential mineral in the staple crop. For instance, it was reported that over-expression of the rice (Oryza sativa L.) nicotianamine synthase 2 (OsNAS2) gene led to an accumulation of higher concentrations of grain Fe, Zn, NA (nicotianamine) and DMA (2'-deoxymugineic acid) in transgenic plants (Beasley et al., 2019). Overexpression of TaVIT2 in wheat and barley resulted in more than twice as much iron in white flour fractions (Connorton et al., 2017). Overexpression of TpSnRK2.10 or TpSnRK2.11 in dwarf Polish wheat significantly increased Fe accumulation in roots and Cd accumulation in roots and shoots (Wang et al., 
2019). Thus, there is an urgent need to explore more about molecular regulations of the Fe accumulationassociated gene expressions in the wheat plant.

Previously, we used EMS (ethylmethylsulfonate) mutagenesis to generate a mutant library from the popular wheat variety 'Luyuan 502' in China (Ma et al. 2021). By screen of the mutant plants, a number of salt-tolerant lines with high crop yield were obtained from the study. Further analysis from the mutant plants in the levels of mineral ions led to identification of two lines with high ferric contents. The presence of the wheat mutant lines with high iron provided a good example to explore changes of endogenous genes responsible for iron accumulation at a transcriptome scale in wheat. In the present study, the iron responsive gene expression, ethylene sensitivity and ethylene signaling-regulated gene expressions were examined in the mutant plants. These results undoubtedly advance our understanding about the molecular regulations of Fe accumulation in the crop.

\section{Materials And Methods}

\section{Plant material and growth condition}

The bread wheat cultivar Luyuan502 (approved by the Chinese National Crop Variety Examination and Approval Committee, 2011) was used for experiments. Seeds of 'Luyuan502' were sterilized in $2 \% \mathrm{NaClO}$ for $10 \mathrm{~min}$, washed in sterile water for $3 \mathrm{~min}$. After washing 5 times, the seeds were placed in the petri dish filled with two layers of sterile filter paper. The surface sterilized seeds were incubated at room temperature. The petri dishes were then incubated in the growth chamber at $25^{\circ} \mathrm{C}$ under 16 -h light/ 8-hour dark for $24 \mathrm{~h}$ or $6 \mathrm{~d}$.

\section{Measurement of iron content}

The surface sterilized seeds were dried in an oven at $65^{\circ} \mathrm{C}$, and then crushed in a grinder. The fine particles of the samples passed through a $1 \mathrm{~mm}$ sieve were collected. $1.00 \mathrm{~g}$ of each sample was mixed in a tube containing $10 \mathrm{~mL} \mathrm{HNO}_{3}$ and $2 \mathrm{~mL} \mathrm{HClO}_{4}$, and placed at $160^{\circ} \mathrm{C}$ overnight until the solution is clear and transparent. $10 \mathrm{~mL}$ deionized water was added into the tube, and mixed well. The iron content of the samples was measured using an inductively coupled plasma emission spectrometer (ICP-OES, PerkinElmer).

\section{Ethylene sensitivity assays}

Surface sterilized seeds in $2 \% \mathrm{NaClO}$ were placed in the petri dish filled with two layers of sterile filter paper and supplemented with sterile ethylene precursor 1-aminocyclopropane-1-carboxylate (ACC) at 300 $\mu \mathrm{M}$. The petri dishes were wrapped in aluminum foil and incubated for $24 \mathrm{~h}$ or $6 \mathrm{~d}$ at room temperature. Ethylene insensitive mutants were picked up from the screen by measuring seedling growth and root length of the etiolated seedlings. Three replicates were taken, and at least 10 seeds were examined in each replicate. 


\section{RT-qPCR analysis}

Total RNA was isolated from $200 \mathrm{mg}$ of plant tissue using a RNAiso Plus (Takara, Japan). cDNA synthesis was performed using M-MLV reverse transcriptase (Takara, Japan). RT-qPCR was performed using Applied Biosystems QuantStudio 5 (Thermo Fisher, USA). Gene-specific primers used for RT-qPCR were provided in Table S1. Sequence information of the wheat genes and their accession numbers were obtained from Wheat@URGI (https://wheat-urgi.versailles.inrae.fr/). The TaACTIN (GenBank: AB181991) was used as the internal control. Each RT-qPCR was carried out with at least three biological replicates in the experiments.

\section{Transcriptome sequencing}

The 2-week-old seedlings of the wheat (Triticum aestivum L.) cultivar 'Luyuan502' and the mutant lines LHFe-1 and LHFe-2 were used for sequencing by Genedenovo Biotechnology Co., Ltd (Guangzhou, China). Three biological replicates were used in each sample. The sequencing library was sequenced by Illumina HiSeq ${ }^{\text {TM }} 2500$, and the libraries were generated using NEBNext ${ }^{\circledR}$ Ultra ${ }^{\text {TM }}$ RNA Library Prep Kit for Illumina ${ }^{\circledR}$ (NEB, USA). The short reads were filtered by an alignment tool (Bowtie2 2.2.8) (Langmead and Salzberg 2012). The obtained reads were then aligned to the reference genome (http://plants.ensembl.org/index.html) (Kim et al. 2013; Bolser et al. 2015). The transcripts were annotated and identified according to Trapnell et al $(2010,2012)$.

The genes with fold change $\geq 2$ and False Discovery Rate (FDR) $<0.05$ were defined as significant Differentially Expressed Genes (DEGs). The Gene Ontology (GO) analysis (http://www.geneontology.org/) and Kyoto Encyclopedia of Genes and Genomes (KEGG) pathways (http://www.genome.jp/kegg/) were constructed, respectively.

\section{Results}

\section{Generation and identification of salt tolerant wheat mutant lines with high iron}

To generate stress-tolerant wheat plants, the bread wheat cultivar 'Luyuan502' was mutagenized by EMS as previously described (Ma, et al. 2021). In brief, the EMS-mutagenized seeds were sown, grown and screened in a saline-alkali soil field $(0.3 \%-0.7 \%$ salinity). A large number of the $\mathrm{M} 1$ lines were harvested. Among them, the salt tolerant wheat lines were selected for further study mainly based on their increased yields by $20 \%-30 \%$ in the $M_{2}-M_{5}$ generations (Ma et al. 2021). Next, the iron contents of the salt tolerant wheat mutant lines were measured. Two lines, LHFe-1 and LHFe-2 generated from 'Luyuan502' possessed remarkably higher iron content than the wild types and other mutant lines (Fig. 1). This observation implied that the genes responsible for iron assimilation, transportation, and accumulation may be altered in the mutant plants.

\section{Transcriptomic profiling of the differentiated expression genes in the wheat mutant lines with high Fe}


The presence of the wheat mutant lines with high iron $(\mathrm{Fe})$ provided a good example to explore the change of endogenous genes responsible for iron accumulation at a scale of transcriptome in wheat. The wheat mutant line LHFe-1 and LHFe-2 were chosen for transcriptome analysis using the wild type 'Luyuan 502' as a control. The 2-week-old seedlings of the wheat plants were collected and used for RNAseq analysis. Based on the comparisons between WT and each of the wheat mutant lines, the transcripts with fold change $\geq 2$ and FDR $<0.05$ were defined as significant DEGs. A venn diagram of DEGs in the comparisons of WT-vs-LHFe-1, WT-vs-LHFe-2 showed 509 DEGs overlapped (Fig. 2;Table S2). The GO and KEGG enrichment analysis indicated that the overlapped DEGs were mostly affected and mainly involved in cellular activities such as catalytic reaction, translation and biologic regulations, biosynthesis of secondary metabolites, metabolism of amino acid, lipid and carbohydrate, and environmental stress signaling pathways (Fig. 2; Table S2). Although a large number of DEGs were picked up from the transcriptome analysis, the key genes responsible for the iron accumulation were largely unknown in the wheat plant.

\section{Expression profiles of iron responsive genes in the wheat mutant lines with high iron}

We next examined the responsive expression levels of the wheat orthologs which were likely related with Fe homeostasis in the other plants. TaYSL 1 (accession number: TraesCS2B01G407900) is the wheat homologue of Arabidopsis AtYSL 1 that encodes a yellow stripe-like protein and participate in seed iron loading via transport of Fe-nicotianamine chelate (Le Jean et al. 2005; Waters et al. 2006; Chu et al. 2010). Analysis of the TaYSL 1 gene expression revealed that the TaYSL 1 transcript levels was obviously induced by iron in the wheat mutant lines, and the levels was higher than that of WT after treatments. In contrast, the iron-induced expression of the TaYSL 1 transcripts was not detected in the wild type (Fig. 3). This result implied that the ability of iron transport in the wheat mutant lines with higher iron was better than the WT. Similarly, TaZIP29, a member of the ZRT/IRT-like protein (ZIP) encoding gene family that may be involved in the transport of non-transferrin bound iron (accession number:

TraesCS6D02G153800) in plants (Bogdan et al. 2016), was highly induced after EDTA-Fe treatment for $24 \mathrm{~h}$. Although endogenous levels of the TaZIP29 transcript were lower in the wheat mutant lines than that of WT, the EDTA-Fe treatment could enhance the expressions of TaZIP29 in most of the samples (Fig. 3), suggesting that the TaZIP29 may participate in the iron accumulation in wheat plants. In addition, it was found that TaFIBP, encoding a ferric ion binding protein (accession number: TraesCS1A01G019900), exhibited a dramatic increase at $24 \mathrm{~h}$ after a low-level period $(0-12 \mathrm{~h})$ among the plants (Fig. 3). Compared to the wild type (WT), the wheat mutant lines (LHFe-1; LHFe-2) showed higher levels of the TaFIBP transcripts at different treatment time points, indicating that high iron contents in the mutant lines may be attributed to the iron-induced TaFIBP expression in wheat plants.

Interestingly, it was found that the wheat homologue TaRTH (accession number: TraesCS5A02G445300) of Arabidopsis AtRTE1 mainly involved in the ETR1 ethylene receptor signaling (Zheng et at. 2017), displayed obvious iron induction when treated with EDTA-Fe for different time in wheat plants (Fig. 3). And, the transcript levels of TaRTH in the wheat mutant lines were mostly higher than that of the wild 
type. These results suggested that ethylene may mediate TaRTH to participate in iron accumulation in wheat plants.

\section{Altered ethylene sensitivity of the wheat mutant seedlings with high iron}

We examined the ethylene sensitivity of the wheat mutant seedlings with high iron. As previously described (Ma et al. 2021), the wheat seeds were soaked and cultivated in the petri dish supplemented with the ethylene precursor ACC at different concentrations $(0,100,200,300$, and $400 \mu \mathrm{M})$ for $48 \mathrm{~h}$, and the longest seedling root lengths were measured. As shown in Figure 4, the seedling root lengths were decreased as ACC concentration increased. Compared to the WT, the seedling root lengths of the wheat mutant lines were obviously longer after the treatment with higher ACC concentrations $(200,300$ and 400 $\mu \mathrm{M})$, indicating that the ethylene sensitivity of the wheat mutant lines with high iron were significantly decreased at seedling stage (Fig. 4a,b).

\section{Expression profiles of ethylene responsive genes in the wheat mutant lines with high iron}

Ethylene sensitivity alteration of the wheat mutant seedlings prompted us to investigate the change of transcript levels among the ethylene signaling-associated genes in the wheat mutant lines. The wheat ETHYLENE RESPONSE FACTOR (ERF) genes (TaERF1, 2, 3, 4 and 6) were chosen for examination of their transcript levels when treated with ACC as previously described (Ma et al. 2021), and the results indicated that transcript levels of the TaERFs were rapidly increased and peaked at $6 \mathrm{~h}$, after then they decreased at $12 \mathrm{~h}$ in the wild type (LY502) (Fig. 5). In order to know whether these TaERFs were affected in the wheat mutant lines with high iron when treated with ACC, the transcript levels of the TaERFs were examined in the seedlings of the mutant lines treated with ACC. As shown in Figure 5, the transcript levels of the TaERFs in LHFe- 1 were also rapidly elevated, but unlike LY502, their peak appeared at $3 \mathrm{~h}$ except for TaERF4. In LHFe-2, the response patterns of the TaERFs were similar to those in LY502 except for TaERF4 (Fig. 5).

In addition to the TaERF1, 2, 3, 4 and 6, two other genes upstream of the ethylene signaling pathway, TaRTH and TaEIN2, were also analyzed. As shown in Figure 5, their response patterns in LY502, LHFe-1 and $L H F e-2$ were similar to those of TaERFs. Curiously, the induction levels of TaRTH in the wheat mutant lines were significantly higher than that in LY502 (Fig. 5). These data suggested that the accumulation of iron in the mutants is likely to be related to the changes of ethylene response in the mutants.

\section{Discussion}

\section{Does iron ion participate in ethylene signaling regulation?}

Ethylene participates in various aspects of plant growth, development, and stress responses, such as seed germination, flowering, sex determination, fruit maturity, senescence, and stress responses (Dubois et al., 2018). It is believed that mineral ions are involved in ethylene response and signaling. In early studies, it was reported that the binding of ethylene to receptors is mediated by a copper cofactor 
(Schaller and Bleecker 1995; Rodriguez et al. 1999; Wang et al. 2006). The Arabidopsis RESPONSE TO ANTAGONIST1 (RAN1) encodes a copper-transporting P-type ATPase and was responsible for the cuprous ion delivery to ethylene receptors (Hirayama et al. 1999; Woeste and Kieber 2000). The loss-offunction ran 1 mutants showed constitutive ethylene responses and hypersensitivity to copper-chelating agents (Hirayama et al. 1999; Woeste and Kieber 2000; Binder et al. 2010). Further studies indicated that RAN1 interacted with ANTIOXIDANT PROTEIN1 (ATX1), loss-of-function of ATX1 showed ethylene hypersensitivity, while overexpression of $A T X 1$ exhibited tolerance to copper (Puig et al. 2007; Shin et al. 2012). In addition to copper, silver also serves as the cofactor for ethylene binding to ETR1 (Beyer 1976; Rodriguez et al. 1999; McDaniel and Binder 2012). In plants, silver is widely used as an inhibitor of ethylene responses. It was reported that ethylene binding to the ETR1 receptor with silver was $\sim 30 \%$ of binding with copper (McDaniel and Binder 2012). Silver may have an effect on ethylene signaling outside of the ethylene binding pocket of the receptors (McDaniel and Binder 2012; Bakshi et al. 2015).

In contrast, little is known whether iron could participate in ethylene response and signaling. In the present study, it was noted that expression of the wheat TaRTH (accession number:

TraesCS5A02G445300), an ortholog of the Arabidopsis AtRTE1 which encodes an activator of the Arabidopsis ETR1 receptor (Resnick et al. 2006, 2008; Zheng et at. 2017), was obviously induced by iron in wheat plants (Fig. 3). In addition, the expression pattern of TaRTH was highly correlated with that of the ferric ion binding protein encoding gene TaFIBP (accession number: TraesCS1A01G019900) and TaZIP29 (accession number: TraesCS6D02G153800) which encodes a ZRT/IRT-like protein (ZIP) involved in the transport of non-transferrin bound iron in plants (Bogdan et al. 2016) (Fig. 3). Although it is unclear whether TaRTH could directly interact with a ferric ion binding protein or an iron transporting protein, it is speculated that iron may mediate TaRTH to take a part in ethylene signaling in wheat plants.

\section{Ethylene is involved in iron accumulation in plants}

In past years, increasing data indicated that the gaseous plant hormone ethylene plays important roles in iron homeostasis in plants. Ethylene is perceived by receptors, and mediates downstream components to transduction into the transcription activator EIN3/EIL1 in the nucleus. EIN3 promotes expressions of the downstream ERFs and other ethylene-responsive genes, thus activating multiple ethylene responses. In Arabidopsis, it was reported that EIN3 can physically interact with and stabilize FIT1, a protein playing a major role in the regulation of Fe acquisition (Lingam et al. 2011), indicating that iron homeostasis could be ethylene-regulated. Later studies provided more evidence demonstrating the involvement of ERFs in iron homeostasis. For example, the Arabidopsis AtERF72 could bind to the promoters of the Fe-deficiency responsive genes such as $I R T 1, H A 2$ and $C L H 1$, and negatively regulate Fe-deficiency responses (Liu et al. 2017). In rice, it was reported that seven $E R F$ genes were transcriptionally regulated under Fe overload stress conditions (dos Santos et al. 2013). More recently, it was observed that a loss-of-function T-DNA insertion allele of ERF95 (erf95-1) confers increased sensitivity to Fe deficiency during early seedling growth of Arabidopsis (Sun et al. 2020). The Arabidopsis ERF95 is a transcriptional activator of FER1, and functioned downstream EIN3 to regulate seed Fe accumulation. In addition, their experiments showed that the abundance of messenger RNAs (mRNAs) from multiple ACS genes encoding 1- 
aminocyclopropane-1-carboxylate (ACC) synthase also increased during seed maturation. It was proposed that ethylene plays an important role in the regulation of Fe accumulation in seed.

In wheat, we showed that high transcript levels of the ERF encoding genes (TaERF1, 2, 3, 4, 6) were detected in the wheat mutant lines with high iron, and the wheat ERF encoding genes were highly induced by exogenous ACC (Fig. 5). In addition, it was observed that ethylene sensitivity of the wheat mutant seedlings with high iron was obviously altered when treated with ACC. All these results suggest that ethylene may participate in regulation of the iron accumulation in wheat. Unfortunately, it is still unclear how ethylene is involved in the Fe homeostasis in wheat plants. Further experiments need to perform to examine whether the wheat ethylene signaling regulator TaRTH could mediate interaction with iron binding protein to regulate iron accumulation.

\section{The genes responsible for iron accumulation in wheat}

Interestingly, the wheat mutant lines with high iron from this study provided a good example to explore changes of the endogenous genes which may be likely responsible for iron accumulation at a scale of transcriptome in wheat. Comparisons in WT-vs-LHFe-1 and WT-vs-LHFe-2 gave 509 DEGs overlapped (Fig. 2; Table S2). Among them, 34 DEGs showing elevated levels were detected in the mutant lines, whereas the rest displayed down-regulated expressions in $L H F e-1$ and $L H F e-2$. The GO and KEGG enrichment analysis indicated that the overlapped DEGs were involved in various processes, including cellular metabolism, catalytic activity, translation, biologic regulation, response to stimulus, and amino acid, lipid and secondary metabolite biosynthesis pathways. All these information provided a foundation for further exploration of the molecular regulations in Fe homeostasis of wheat.

Meanwhile, expression analysis of the known genes responsible for Fe homeostasis in wheat strongly supports that the ability of iron accumulation in the wheat mutant lines with higher iron was better than the WT (Fig. 3). For example, wheat TaFIBP encoded a ferric ion binding protein (accession number: TraesCS1A01G019900), and exhibited a dramatic increase at $24 \mathrm{~h}$ when treated with $1.5 \mathrm{mM}$ EDTA-Fe (Fig. 3). Compared to the wild type (WT), the wheat mutant lines (LHFe-1; LHFe-2) showed higher levels of the TaFIBP transcripts at different treatment time points. As shown in Figure 3, a similar response was observed for the wheat TaZIP29 which encodes a member of the ZRT/IRT-like protein (ZIP) and may participate in the transport of non-transferrin bound iron (accession number: TraesCS6D02G153800) in plants (Bogdan et al. 2016). Moreover, this study also analyzed the iron response of TaYSL 1 (accession number: TraesCS2B01G407900), a wheat homologue of AtYSL 1 that encodes the Arabidopsis yellow stripe-like protein and participate in seed iron loading via transport of Fe-nicotianamine chelate (Le Jean et al. 2005; Waters et al. 2006; Chu et al. 2010), and its expression was obviously induced by iron and the transcript levels were higher than that of WT (Fig. 3), suggesting that the iron transport in the wheat mutant lines with higher iron was better than the WT.

\section{Declarations}

\section{Acknowledgments}


This work was financially supported by Shandong Agricultural Variety Project (2019LZGC015) to CHD and Shandong Provincial Natural Science Foundation (ZR2021MC020) to YY.

\section{Author Contributions}

$\mathrm{NL}, \mathrm{YL}, \mathrm{JS}$, and XS performed the experiments. YY did data analysis and wrote a version of the manuscript. CHD designed the experiments and did revision of the manuscript.

All authors have read and approved the manuscript. We declared that the authors of this paper have no conflict of interest.

\section{References}

1. Bakshi A, Wilson RL, Lacey RF, Kim H, Wuppalapati SK, Binder BM (2015) Identification of regions in the receiver domain of the Ethylene RESPONSE1 ethylene receptor of Arabidopsis important for functional divergence. Plant Physiol 169:219-232

2. Beasley JT, Bonneau JP, Sánchez-Palacios JT, Moreno-Moyano LT, Callahan DL, Tako E, Glahn RP, Lombi E, Johnson AAT (2019) Metabolic engineering of bread wheat improves grain iron concentration and bioavailability. Plant Biotechnol J 17(8):1514-1526

3. Beyer EM (1976) A potent inhibitor of ethylene action in plants. Plant Physiol 58:268-371

4. Bleecker AB, Estelle MA, Somerville C, Kende H (1988) Insensitivity to ethylene conferred by a dominant mutation in Arabidopsis thaliana. Science 241(4869):1086-1089

5. Binder BM, Rodriguez Fl, Bleecker AB (2010) The copper transporter RAN1 is essential for biogenesis of ethylene receptors in. Arabidopsis J Biol Chem 48:37263-37270

6. Bogdan AR, Miyazawa M, Hashimoto K, Tsuji Y (2016) Regulators of Iron Homeostasis: New Players in Metabolism, Cell Death, and Disease. Trends Biochem Sci 41(3):274-286

7. Bolser DM, Kerhornou A, Walts B, Kersey P (2015) Triticeae resources in ensembl plants. Plant Cell Physiol 56(1):e3

8. Breiman A, Graur B (1995) Wheat evolution. Isr J Plant Sci 43:85-98

9. Briat JF, Dubos C, Gaymard F (2015) Iron nutrition, biomass production, and plant product quality. Trends Plant Sci 20:33-40

10. Chu HH, Chiecko J, Punshon T, Lanzirotti A, Lahner B, Salt DE, Walker EL (2010) Successful reproduction requires the function of Arabidopsis Yellow Stripe-Like1 and Yellow Stripe-Like3 metalnicotianamine transporters in both vegetative and reproductive structures. Plant Physiol 154:197210

11. Connorton JM, Jones ER, Rodríguez-Ramiro I, Fairweather-Tait S, Uauy C, Balk J (2017) Wheat Vacuolar Iron Transporter TaVIT2 Transports Fe and Mn and Is Effective for Biofortification. Plant Physiol 174(4):2434-2444

12. Curie C, Briat JF (2003) Iron transport and signaling in plants. Annu Rev Plant Biol 54:183-206 
13. Dos Santos RS, Kruger MM, Pegoraro C, Madabula FP, da Maia LC, Rombaldi CV, de Oliveira AC (2013) Transcriptional regulation of seven ERFs in rice under oxygen depletion and iron overload stress. Tropic Plant Biol 6:16-25

14. Dong W, Ai X, Xu F, Quan T, Liu S, Xia G (2012) Isolation and characterization of a bread wheat salinity responsive ERF transcription factor. Gene 511(1):38-45

15. Dubois $M$, Van den Broeck L, Inze D (2018) The pivotal role of ethylene in plant growth. Trends Plant Sci 23(4):311-323

16. Giehl R, Lima J, von Wirén N (2012) Localized iron supply triggers lateral root elongation in Arabidopsis by altering the AUX1-mediated auxin distribution. Plant Cell 24:33-49

17. Hansch R, Mendel RR (2009) Physiological functions of mineral micronutrients (Cu, Zn, Mn, Fe, Ni, Mo, B, Cl). Curr Opin Plant Biol 12:259-266

18. Hell R, Stephan UW (2003) Iron uptake, trafficking and homeostasis in plants. Planta 210:541-551

19. Hirayama T, Kieber JJ, Hirayama N, Kogan M, Guzman P, Nourizadeh S, Alonso JM, Dailey WP, Dancis A, Ecker JR (1999) Responsive-to-antagonist 1, a Menkes/Wilson disease-related copper transporter, is required for ethylene signaling in Arabidopsis. Cell 97:383-393

20. Ivanov R, Brumbarova T, Bauer P (2012) Fitting into the harsh reality: regulation of iron-deficiency responses in dicotyledonous plants. Mol Plant 5:27-42

21. Jiang C, Belfield EJ, Cao Y, Smith JA, Harberd N (2013) An Arabidopsis soil-salinity-tolerance mutation confers ethylene-mediated enhancement of sodium/potassium homeostasis. Plant Cell 25(9):3535-3552

22. Jin C, Du S, Shamsi I, Luo B, Lin X (2011) NO synthase-generated NO acts downstream of auxin in regulating Fe-deficiency-induced root branching that enhances Fe-deficiency tolerance in tomato plants. J Exp Bot 62:3875-3884

23. Kim D, Pertea G, Trapnell C, Pimentel H, Kelley R, Salzberg SL (2013) TopHat2: accurate alignment of transcriptomes in the presence of insertions, deletions and gene fusion. Genome Biol 14(4):R36

24. Kobayashi T, Itai RI, Senoura T, Oikawa T, Ishimaru Y, Ueda M (2016) Jasmonate signaling is activated in the very early stages of iron deficiency responses in rice roots. Plant Mol Biol 91:533547

25. Kobayashi T, Nishizawa NK (2012) Iron uptake, translocation, and regulation in higher plants. Annu Rev Plant Biol 63:132-152

26. Langmead B, Salzberg SL (2012) Fast gapped-read alignment with Bowtie2. Nat Methods 9(4):357359

27. Le Jean M, Schikora A, Mari S, Briat JF, Curie C (2005) A loss-of-function mutation in AtYSL1 reveals its role in iron and nicotianamine seed loading. Plant $\mathrm{J}$ 44:769-782

28. Liu K, Yue R, Yuan C, Liu J, Zhang L, Sun T, Yang Y, Tie S, Shen C (2015) Auxin signaling is involved in iron deficiency-induced photosynthetic inhibition and shoot growth defect in rice (Oryza sativa L.). J Plant Biol 58:391-401 
29. Liu W, Li QW, Wang Y, Wu T, Yang YF, Zhang XZ, Han ZH, Xu XF (2017) Ethylene response factor AtERF72 negatively regulates Arabidopsis thaliana response to iron deficiency. Biochem Biophys Res Commun 491:862-868

30. Lingam S, Mohrbacher J, Brumbarova T, Potuschak T, Fink-Straube C, Blondet E, Genschik P, Bauer P (2011) Interaction between the bHLH transcription factor fit and ethylene insensitive3/ethylene insensitive3-like1 reveals molecular linkage between the regulation of iron acquisition and ethylene signaling in Arabidopsis. Plant Cell 23:1815-1829

31. Ma Q, Zhou HJ, Sui XY, Su CX, Yu YC, Yang HB, Dong CH (2021) Generation of new salt-tolerant wheat lines and transcriptomic exploration of the responsive genes to ethylene and salt stress. Plant Growth Regul 94:33-48

32. Matsuoka K, Furukawa J, Bidadi H, Asahina M, Yamaguchi S, Satoh S (2014) Gibberellin-induced expression of Fe-uptake-related genes in Arabidopsis. Plant Cell Physiol 55:87-98

33. McDaniel BK, Binder BM (2012) Ethylene receptor 1 (ETR1) is sufficient and has the predominant role in mediating inhibition of ethylene responses by silver in Arabidopsis thaliana. J Biol Chem 287(31):26094-26103

34. Morgan PW, Drew MC (1997) Ethylene and plant responses to stress. Physiol Plant 100:620-630

35. Peng J, Li Z, Wen X, Li W, Shi H, Yang L, Zhu H, Guo H (2014) Salt induced stabilization of EIN3/EIL1 confers salinity tolerance by deterring ROS accumulation in Arabidopsis. PLoS Genet 10:e1004664

36. Puig S, Mira H, Dorcey E, Sancenon V, Andres-Colas N, Garcia-Molina A, Burkhead JL, Gogolin KA, Abdel-Ghany SE, Thiele DJ, Ecker JR, Pilon M, Penarrubia L (2007) Higher plants possess two different types of ATX1-like copper chaperones. Biochem Biophys Res Commun 354(2):385-390

37. Qi Y, Wang S, Shen C, Zhang S, Chen Y, Xu Y, Liu Y, Wu Y, Jiang D, OsARF12 (2012) A transcription activator on auxin response gene regulates root elongation and affects iron accumulation in rice (Oryza sativa). New Phytol 193:109-120

38. Rai V, Sanagala R, Sinilal B, Yadav S, Sarkar AK, Dantu PK, Jain A (2015) Iron Availability Affects Phosphate Deficiency-Mediated Responses, and Evidence of Cross-Talk with Auxin and Zinc in Arabidopsis. Plant Cell Physiol 56(6):1107-1123

39. Resnick JS, Wen CK, Shockey JA, Chang C (2006) REVERSION-TO-ETHYLENE SENSITIVITY1, a conserved gene that regulates ethylene receptor function in Arabidopsis. Proc Natl Acad Sci USA 103:7917-7922

40. Resnick JS, Rivarola M, Chang C (2008) Involvement of RTE1 in conformational changes promoting ETR1 ethylene receptor signaling in Arabidopsis. Plant J 56:423-431

41. Rodriguez FI, Esch JJ, Hall AE, Binder BM, Schaller GE, Bleecker AB (1999) A copper cofactor for the ethylene receptor ETR1 from Arabidopsis. Science 283:996-998

42. Roschzttardtz H, Conejero G, Divol F, Alcon C, Verdeil JL, Curie C, Mari S (2013) New insights into Fe localization in plant tissues. Front Plant Sci 4:350

43. Romera FJ, Alcántara E (2004) Ethylene involvement in the regulation of Fe deficiency stress responses by Strategy I plants. Funct Plant Biol 31:315-328 
44. Romera FJ, Alcantara E, De La Guardia MD (1999) Ethylene production by Fe-deficient roots and its involvement in the regulation of Fe-deficiency stress responses by Strategy I plants. Ann Botany 83:51-55

45. Römheld V, Marschner H (1986) Evidence for a specific uptake system for iron phytosiderophore in roots of grasses. Plant Physiol 80:175-180

46. Rong W, Qi L, Wang A, Ye X, Du L, Liang H, Xin Z, Zhang Z (2014) ERF transcription factor TaERF3 promotes tolerance to salt and drought stresses in wheat. Plant Biotechnol J 12:468-479

47. Schaller GE, Bleecker AB (1995) Ethylene-binding sites generated in yeast expressing the Arabidopsis ETR1 gene. Science 270:1809-1811

48. Shin LJ, Lo JC, Yeh KC (2012) Copper chaperone antioxidant protein 1 is essential for copper homeostasis. Plant Physiol 159(3):1099-1110

49. Stacey MG, Patel A, McClain WE, Mathieu M, Remley M, Rogers EE, Gassmann W, Blevins DG, Stacey G (2008) The Arabidopsis AtOPT3 protein functions in metal homeostasis and movement of iron to developing seeds. Plant Physiol 146:589-601

50. Sun Y, Li JQ, Yan JY, Yuan JJ, Li GX, Wu YR, Xu JM, Huang RF, Harberd NP, Ding ZJ, Zheng SJ (2020) Ethylene promotes seed iron storage during Arabidopsis seed maturation via ERF95 transcription factor. J Integr Plant Biol 62(8):1193-1212

51. Trapnell C, Roberts A, Goff L, Pertea G, Kim G, Kelley DR, Pimentel H, Salzberg SL, Rinn JL, Pachter L (2012) Differential gene and transcript expression analysis of RNA-seq experiments with TopHat and Cufflinks. Nat Protoc 7(3):562-578

52. Trapnell C, Williams BA, Pertea G, Mortazavi A, Kwan G, van Baren MJ, Salzberg SL, Wold BJ, Pachter $L$ (2010) Transcript assembly and quantification by RNA-seq reveals unannotated transcripts and isoform switching during cell differentiation. Nat Biotechnol 28:511-515

53. van Loon LC, Geraats BP, Linthorst HJ (2006) Ethylene as a modulator of disease resistance in plants. Trends Plant Sci 11(4):184-191

54. Wang B, Wei H, Xue Z, Zhang WH (2017) Gibberellins regulate iron deficiency-response by influencing iron transport and translocation in rice seedlings (Oryza sativa). Ann Bot 119(6):945-956

55. Wang BL, Li G, Zhang WH (2015) Brassinosteroids are involved in Fe homeostasis in rice (Oryza sativa L.). J Exp Bot 66:2749-2761

56. Wang H, Wang F, Zheng F, Wang L, Pei H, Dong CH (2016) Ethylene-insensitive mutants of Nicotiana tabacum exhibit drought stress resistance. Plant Growth Regul 79(1):107-117

57. Wang R, Wang C, Yao Q, Xiao X, Fan X, Sha L, Zeng J, Kang H, Zhang H, Zhou Y, Wang Y The polish wheat (Triticum polonicum L.) (2019) TpSnRK2.10 and TpSnRK2.11 meditate the accumulation and the distribution of $\mathrm{cd}$ and Fe in transgenic Arabidopsis plants.BMC Genomics20(1):210

58. Wang WY, Esch JJ, Shiu SH, Agula H, Binder BM, Chang C, Patterson SE, Bleecker AB (2006) Identification of important regions for ethylene binding and signaling in the transmembrane domain of the ETR1 ethylene receptor of Arabidopsis. Plant Cell 18:3429-3442 
59. Waters BM, Chu HH, Didonato RJ, Roberts LA, Eisley RB, Lahner B, Salt DE, Walker EL (2006) Mutations in Arabidopsis yellow stripe-like1 and yellow stripe-like3 reveal their roles in metal ion homeostasis and loading of metalions in seeds. Plant Physiol 141:1446-1458

60. Woeste KE, Kieber JJ (2000) A strong loss-of-function mutation in RAN1 results in constitutive activation of the ethylene response pathway as well as a rosette-lethal phenotype. Plant Cell 12:443455

61. Xu Y, Zhang S, Guo H, Wang S, Xu L, Li C, Qian Q, Chen F, Geisler M, Qi Y, Jiang A (2014) OsABCB14 functions in auxin transport and iron homeostasis in rice (Oryza sativa L.). Plant J 79:106-117

62. Xu ZS, Xia LQ, Chen M, Cheng XG, Zhang RY, Li LC, Zhao YX, Lu Y, Ni ZY, Liu L, Qiu ZG (2007) Isolation and molecular characterization of the Triticum aestivum $\mathrm{L}$. ethylene-responsive factor 1 (TaERF1) that increases multiple stress tolerance. Plant Mol Biol 65(6):719-732

63. Yang C, Lu X, Ma B, Chen SY, Zhang JS (2015) Ethylene signaling in rice and Arabidopsis: conserved and diverged aspects. Mol Plant 8(4):495-505

64. Yang C, Ma B, He SJ, Xiong Q, Duan KX, Yin CC, Chen H, Lu X, Chen SY, Zhang JS (2015) Maohuzi6/ethylene insensitive3-like1 and ethylene insensitive3-like2 regulate ethylene response of roots and coleoptiles and negatively affect salt tolerance in rice. Plant Physiol 169(1):148-165

65. Yang J, Chen W, Chen L, Qin C, Jin C, Shi Y, Shi Y, Zheng S (2013) The 14-3-3 protein GENERAL REGULATORY FACTOR11 (GRF11) acts downstream of nitric oxide to regulate iron acquisition in Arabidopsis thaliana. New Phytol 197:815-824

66. Yu Y, Qi Y, Xu J, Dai X, Chen J, Dong CH, Xiang F (2021) Arabidopsis WRKY71 regulates ethylenemediated leaf senescence by directly activating EIN2, ORE1 and ACS2 genes. Plant J 107(6):18191836

67. Zhang L, Liu P, Wu J, Qiao L, Zhao G, Jia J, Gao L, Wang J (2020) Identification of a novel ERF gene, TaERF8, associated with plant height and yield in wheat. BMC Plant Biol 20:263

68. Zheng F, Cui X, Rivarola M, Gao T, Chang C, Dong CH (2017) Molecular association of Arabidopsis RTH with its homolog RTE1 in regulating ethylene signaling. J Exp Bot 68(11):2821-2832

\section{Figures}




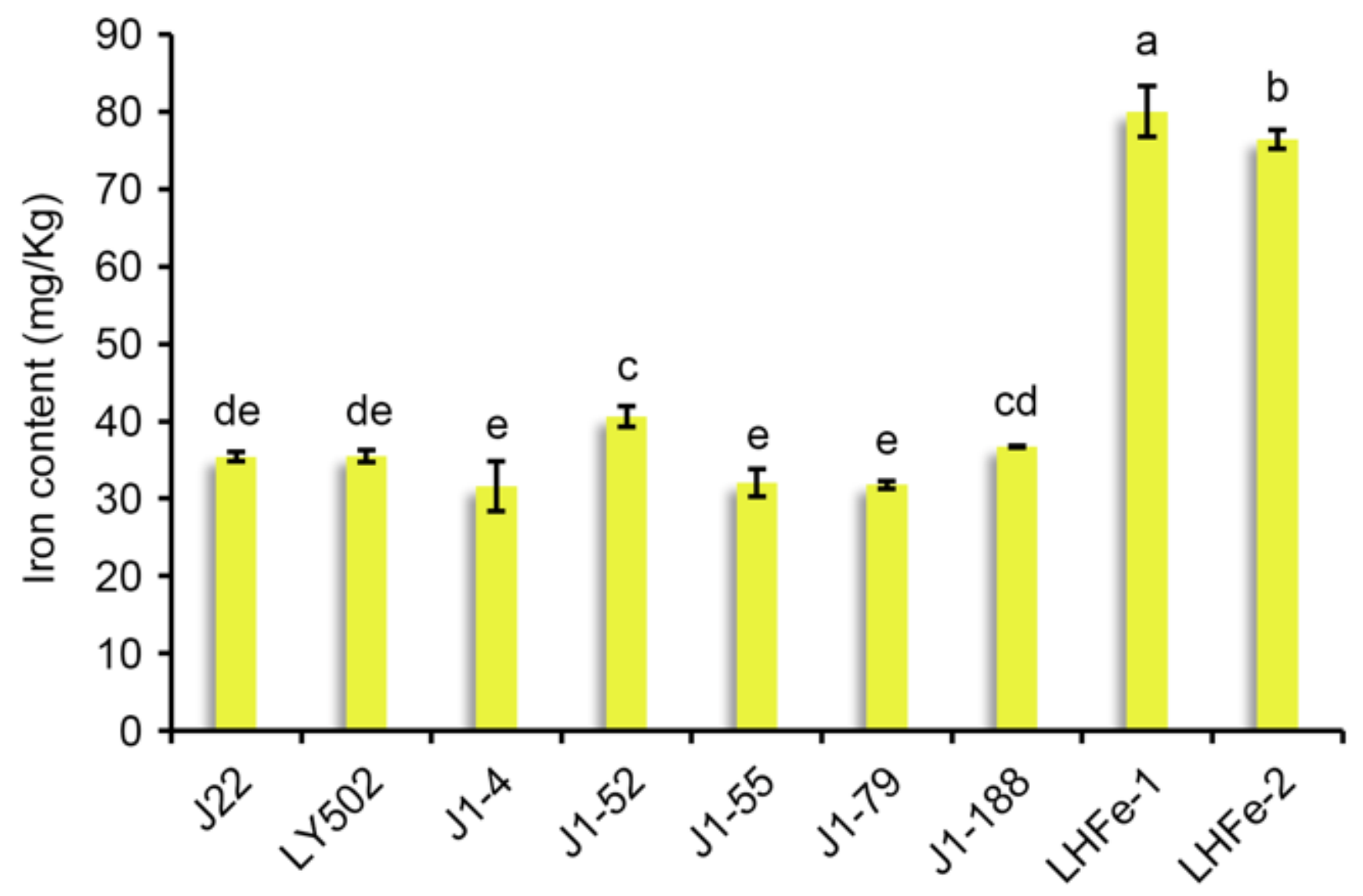

Figure 1

The iron contents of the wild type and salt tolerant wheat mutant lines.

The seeds of different wheat lines were directly used to measure the iron contents. Error bars represented SD $(n=3)$. Different lowercase letters above the columns indicated the significant differences by Tukey's test $(P<0.05)$.

\section{Figure 2}

Comparisons of LY502-vs-LHFe-1 and LY502-vs-LHFe-1.

(A)The venn diagrams of DEGs in the comparisons of LY502-vs-LHFe-1 and LY502-vs-LHFe-1. (B) GO enrichment analysis of the DEGs in the comparisons. (C) KEGG enrichment analysis of the DEGs in the comparisons. 

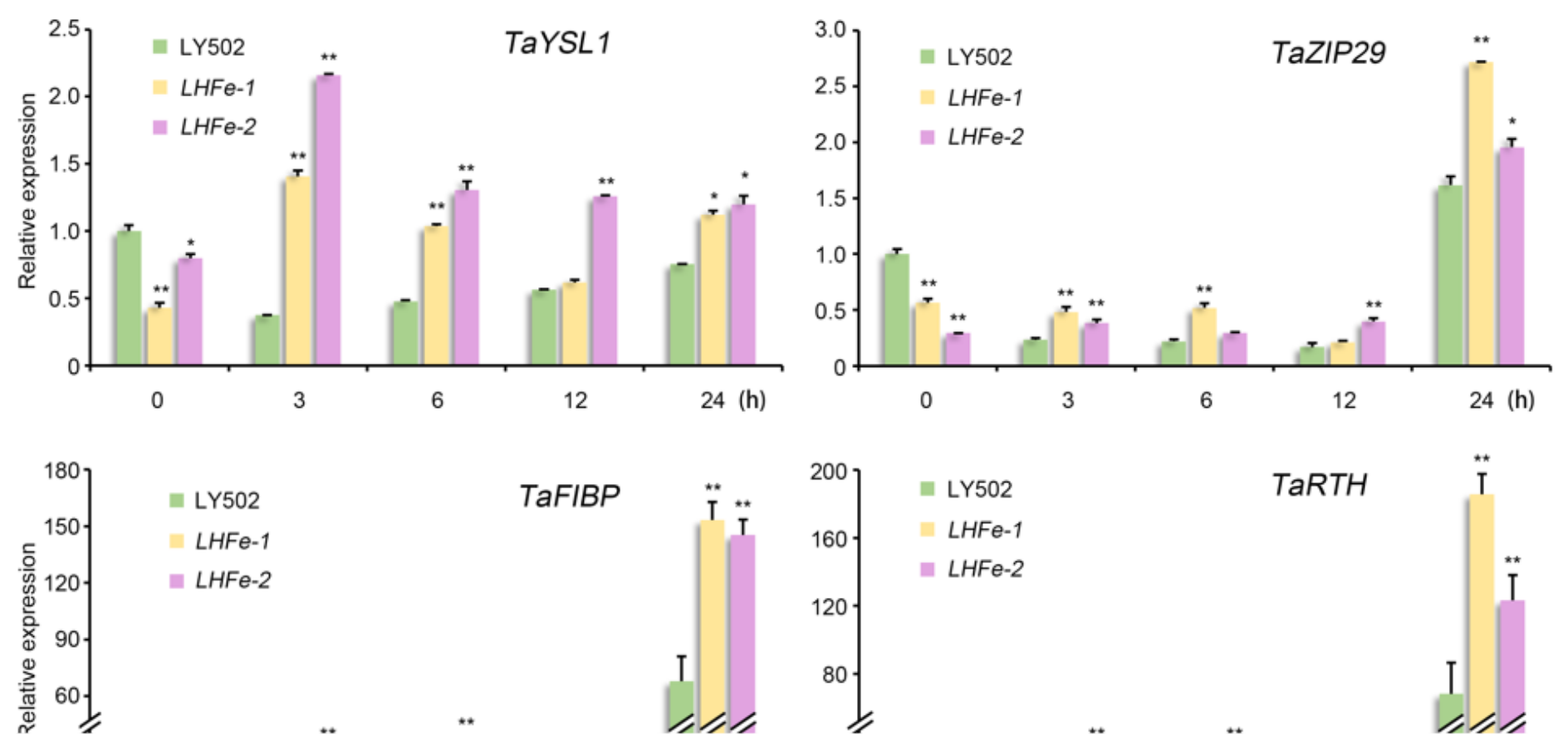

\section{Figure 3}

Expression profiles of iron responsive genes in LY502, LHFe-1 and LHFe-2.

Two-week old wheat seedlings were treated with 1.5 mM EDTA-Fe for different times as indicated and then used for RNA isolation and gene transcript levels detection. TaACTIN was used as an internal control. Error bars represented SD $(n=3)$. The significant differences between the wild type and mutant wheats in each group were valued by Student's t test $(* * P<0.01, * P<0.05)$. 
A

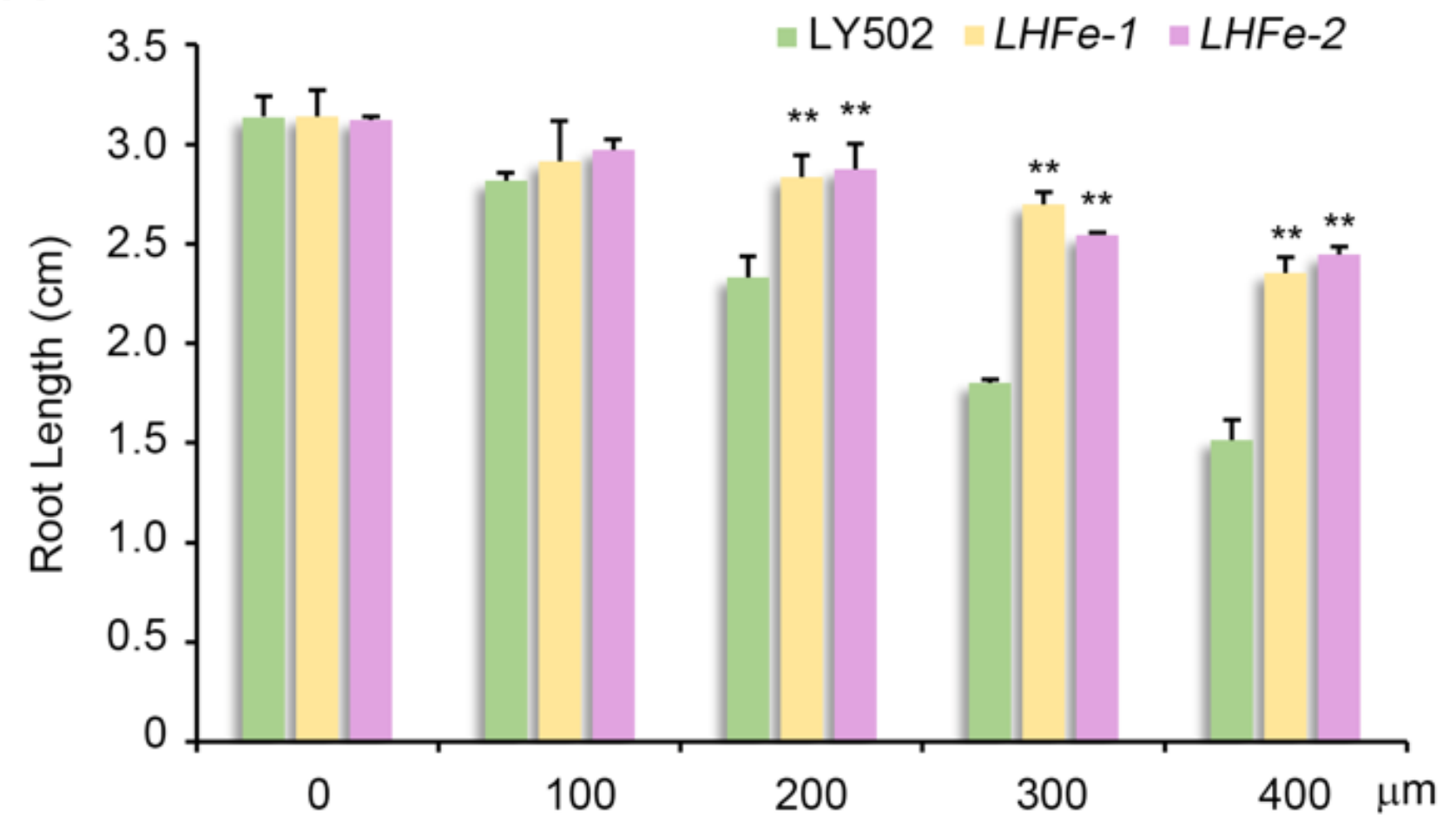

B

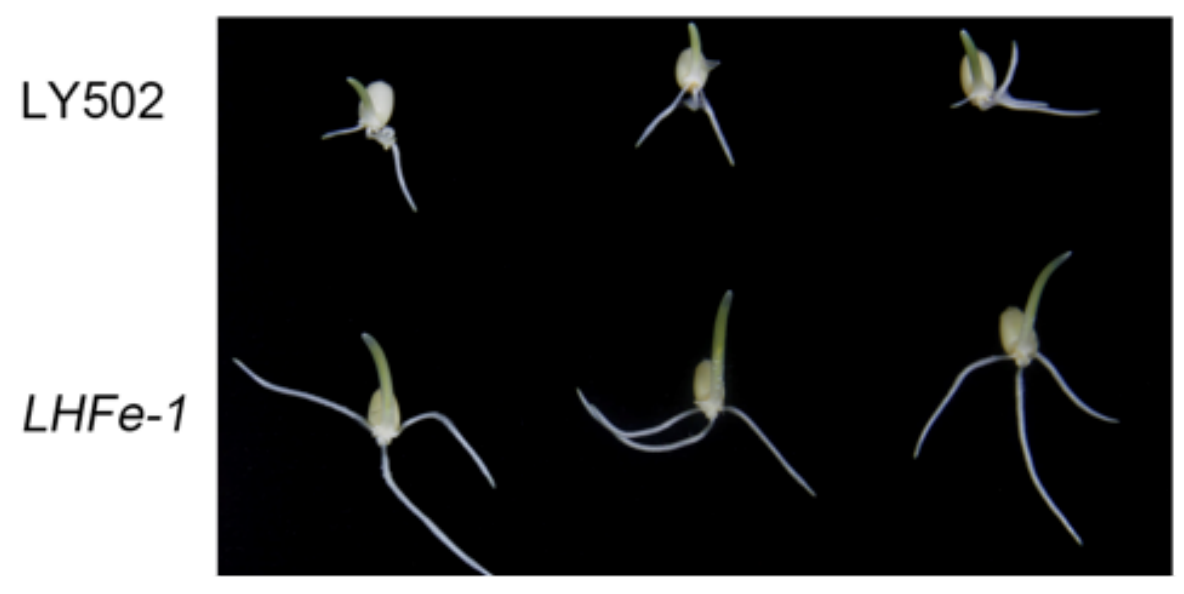

Figure 4

The wheat mutant lines with high iron were insensitive to ethylene.

(A) The root length of LY502, LHFe-1 and LHFe-2 treated with ACC at different concentrations. Error bars represented SD $(n=3)$. The significant differences between the wild type and mutant wheats in each 
group were valued by Student's t test ( $\left.{ }^{\star \star} P<0.01,{ }^{\star} P<0.05\right)$. (B) The root phenotypes of LY502, LHFe-1 and LHFe-2 treated with $300 \mu \mathrm{M}$ ACC under dark conditions.
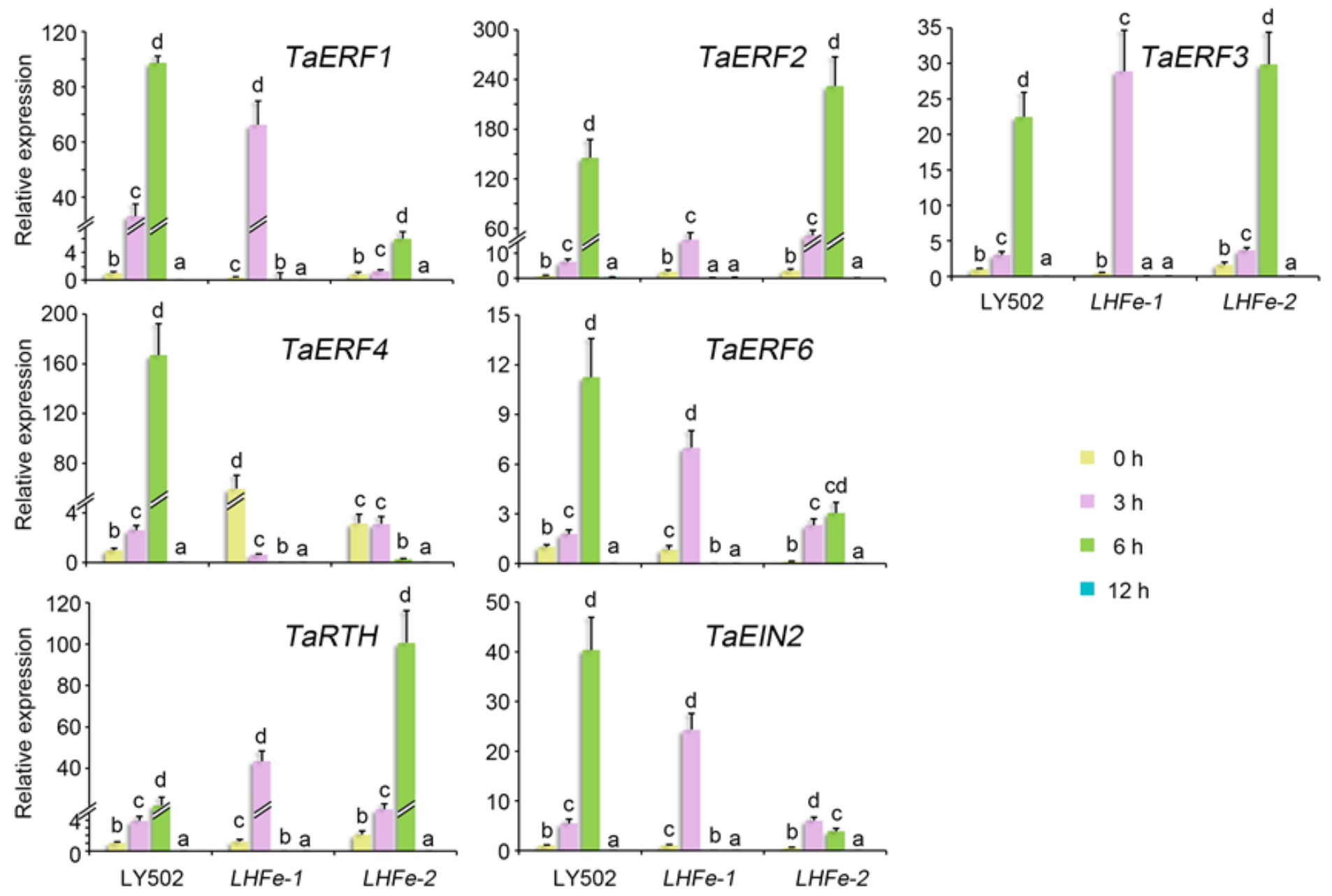

\section{Figure 5}

Expression profiles of Ethylene responsive genes in LY502, LHFe-1 and LHFe-2.

Two-week old wheat seedlings were treated with $300 \mu \mathrm{M}$ ACC for different times as indicated and then used for RNA isolation and gene transcript levels detection. TaACTIN was used as an internal control. Error bars represented SD $(n=3)$. Different lowercase letters above the columns indicated the significant differences in each line by Tukey's test $(P<0.05)$.

\section{Supplementary Files}

This is a list of supplementary files associated with this preprint. Click to download. 
- TableS1Primers.doc

- Tables2.xls 